Juliane Finger

\title{
Gestaltung des digitalen Kulturwandels zu Open Access im Programm Hamburg Open Science
}

\section{Ein Erfahrungsbericht}

Die Digitalisierung eröffnet zahlreiche neue Möglichkeiten für die Kommunikation von Forschungsergebnissen. Trotz vieler bekannter Vorteile von Open Access (OA) gibt es aber immer noch Hinderungsgründe für Forschende, ihre Werke auch in dieser Form zu veröffentlichen. Im Zeitraum 2016 bis 2020 erschien nur etwa die Hälfte aller wissenschaftlichen Artikel in Deutschland im Open Access. ${ }^{1}$

Dieser Beitrag beschäftigt sich mit dem Projekt ,Gestaltung des digitalen Kulturwandels‘ (im Folgenden kurz: Projekt Kulturwandel) des Programms ,Hamburg Open Science' (HOS). Ziel dieses Projekts ist es, den digitalen Wandel hin zu mehr OA zu unterstützen. Hinter dem Begriff „Kulturwandel“ steht im Projekt die Beobachtung, dass jede Fachdisziplin, jede*r Wissenschaftler*in spezifische Strategien bzw. Kulturen zur Planung, Umsetzung und Veröffentlichung von Forschungsergebnissen hat. Um diese tradierten oder persönlichen Strategien zu verändern und analog zu den technischen Möglichkeiten weiterzuentwickeln, bedarf es eines Kulturwandels.

Das Projekt unterstützt diesen Wandel, indem es eine Reflexion des bisher praktizierten Verhaltens anregt. Außerdem wird Forschenden mit Beratungsangeboten dabei geholfen, ihre Strategien anzupassen. Der Forschungsprozess soll zudem durch Serviceangebote vereinfacht werden. Das Projekt Kulturwandel ist wie oben erwähnt Teil des hochschulübergreifenden Programms HOS. In diesem geht es darum, Forschungsergebnisse und -daten von Forschungseinrichtungen in Hamburg, die mit Mitteln der staatlichen Forschungsförderung entstanden sind, frei zugänglich und zusammen mit weiteren Informationen zur Wissenschaft in Hamburg einfach auffindbar zu machen. Der Fokus liegt nicht nur auf OA-Publikationen, sondern auch auf weiteren Gesichtspunkten offener Wissenschaft (Open Science), wie dem Teilen von Forschungsdaten oder -informationen. Daher werden im Projekt Kulturwandel generell alle Aspekte offener Wissenschaft in den Blick genommen. In diesem Beitrag werden jedoch primär jene Punkte herausgegriffen, welche der Unterstützung von OA-Publikationen dienen.

Im Folgenden werden zunächst das Gesamtprogramm HOS und die Rolle des Projekts Kulturwandel innerhalb dieses Programms kurz vorgestellt. Anschließend werden die Projektziele und das konkrete Vorgehen genauer dargestellt. Die praktischen Erfahrungen bei der Unterstützung des Kulturwandels zu Open Access werden am Beispiel der Arbeit an der HafenCity Universität Hamburg (HCU) in den Jahren 2018 und 2019 illustriert. Der Beitrag schließt mit einem Blick auf bisherige Erfolgsfaktoren und in die Zukunft. 


\section{Hintergrund: Das Programm ,Hamburg Open Science‘}

Das Programm HOS wird von der Behörde für Wissenschaft, Forschung und Gleichstellung in Hamburg mit einer Laufzeit von drei Jahren (2018-2020) finanziert. Zusammen mit dem auf die Lehre ausgerichteten Programm ,Hamburg Open Online University (HOOU) und der Förderung der IT-Grundlagenforschung ,ahoi.Digital ' bildet HOS den Rahmen für die digitale Transformation der Hamburger Hochschulen. Dahinter steht der politische Auftrag, öffentlich finanzierte Forschung auch öffentlich zugänglich zu machen. Das Programm baut auf dem politischen Ziel der Entwicklung einer Open-Access-Strategie im Koalitionsvertrag von SPD und Grünen von 2015 auf. 2 Beteiligt sind alle staatlichen Hamburger Hochschulen, das Universitätsklinikum Eppendorf und die Staats- und Universitätsbibliothek Hamburg Carl von Ossietzky. Der Begriff ,Open Science‘ steht dabei für die Idee, durch Digitalisierung die Kommunikation über Forschung und das Teilen von Forschungsergebnissen so offen wie möglich auszugestalten und den Zugang zu vereinfachen.

HOS hat eine starke Infrastrukturkomponente, d.h. es werden technische Systeme für die offene Wissenschaft geschaffen. Ergänzend ermöglicht eine Webplattform den Zugang zu Hamburger Forschungsergebnissen für die interessierte Öffentlichkeit. Insgesamt umfasst es acht verschiedene Teilprojekte (s. Abb. 1): Aufbau bzw. Weiterentwicklung von Repositorien (Projekt Open Access Repositorien), Publikation von Forschungsdaten (Projekt Forschungsdatenmanagement), Publikation von dreidimensionalen und audiovisuellen Forschungsdaten (Projekt 3D / AV), Informationen über Forschungstätigkeiten (Projekt Forschungsinformationssysteme), eine Webplattform für die Darstellung der Hamburger Forschung (Projekt Schaufenster), ein hochschulübergreifendes Archivspeichersystem für Forschungsdaten (Projekt Archivdatenspeicher), Weiterentwicklung von kollaborativen Publikationsprozessen (Projekt Modernes Publizieren), sowie das Projekt Kulturwandel als die Anwendung der technischen Systeme förderndes Querschnittsprojekt.

\begin{tabular}{l|l|}
\begin{tabular}{l} 
Die Projekte von Hamburg Open Science \\
\hline Forschungsdatenmanagement
\end{tabular} & Forschungsinformationssysteme \\
\hline Open Access Repositorien & Archivdatenspeicher \\
\hline Modernes Publizieren & Webplattform (Schaufenster) \\
\hline 3D / AV & Gestaltung des digitalen Kulturwandels \\
\hline
\end{tabular}

Abb. 1: Die Projekte im Programm HOS. 
Im Projekt Kulturwandel sollen Forschende unterstützt werden, die Chancen der Digitalisierung zu nutzen und Forschungsergebnisse offen mit anderen zu teilen. Es war 2018 zunächst dem Programmmanagement von HOS zugeordnet. Daran beteiligt waren neben dem Programmmanager auch Mitarbeitende der HCU sowie der Hochschule für Angewandte Wissenschaften Hamburg (HAW). Seit 2019 ist das Projekt eigenständig unter Beteiligung von Mitarbeiter*innen aller an HOS teilnehmenden Einrichtungen.

Das Konzept von HOS ist die Entwicklung individueller, passgenauer OpenScience-Lösungen für die partizipierenden Einrichtungen. Darüber hinaus werden in der hochschulübergreifenden Zusammenarbeit sowie im Austausch zwischen den Hochschulen Synergieeffekte hergestellt und zusätzlich zu der lokalen Projektarbeit auch gemeinsame Vorhaben verwirklicht. Über verschiedene Schnittstellen gibt es einen kontinuierlichen Austausch zwischen den einzelnen Projekten von HOS. Für das Thema OA finden sich solche Schnittstellen zum Beispiel beim Projekt Modernes Publizieren und natürlich beim Projekt Open-Access-Repositorien.

\section{Das Projekt ,Gestaltung des digitalen Kulturwandels‘}

Für Forschende bedeutet es eine Herausforderung, wenn sie von bekannten Prozessen, z. B. bei der Frage wo und wie sie ihre Forschungsergebnisse veröffentlichen, abweichen und neue Praktiken offener Wissenschaft in ihren Forschungsalltag integrieren möchten. Um diesen ,Kulturwandel` zu unterstützen, verfolgt das Querschnittsprojekt folgende drei übergeordneten Ziele:

1. Das Bewusstsein für Open Science soll bei den Forschenden gestärkt werden.

2. Es sollen Maßnahmen entwickelt werden, um Forschende bei der praktischen Umsetzung von Open Science zu unterstützen. Dabei sollen Forschende weiterhin den Fokus auf ihre eigentliche Forschungsarbeit legen können.

3. Mit dem Projekt soll die praktische Anwendung der im Rahmen des Programms Hamburg Open Science entwickelten technischen Systeme unterstützt werden.

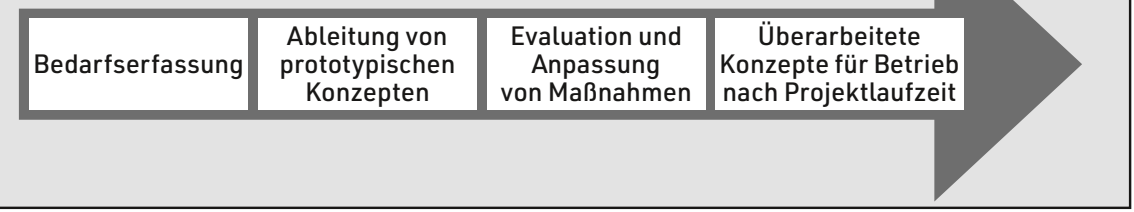

Abb. 2: Geplantes Vorgehen im Projekt Kulturwandel. 
Das Projekt umfasst vier geplante Phasen: In der ersten werden Erhebungen zum Status Quo der Kenntnisse und Einstellungen von Forschenden zu Open Access und Open Science durchgeführt. Ausgehend von den Ergebnissen dieser Bedarfserfassung werden in der zweiten Projektphase prototypische Konzepte und Maßnahmen abgeleitet. In der dritten Projektphase werden diese Maßnahmen evaluiert und angepasst. In der vierten Phase werden auf der Basis der vorangegangenen Arbeiten Konzepte für die Zeit nach Projektende (ab 2021) entwickelt.

\section{Fallbeispiel HafenCity Universität Hamburg}

Im Folgenden werden das Vorgehen und die Erfahrungen aus den ersten Projektphasen zum Thema Open Access am Beispiel der HCU genauer dargestellt. Die HCU ist mit etwa 170 Beschäftigten im wissenschaftlichen Bereich (Stand: Dezember 2018) eine vergleichsweise kleine Hochschule. In den verschiedenen Fachgebieten arbeiten Forschende zu Zukunftsfragen des Bauens sowie zur Metropolenentwicklung und begleiten urbane Transformationsprozesse. Die akademische Tätigkeit ist stark von interund transdisziplinärer Zusammenarbeit geprägt. Innerhalb der HCU ist das Projekt Kulturwandel organisatorisch an die Bibliothek angegliedert.

\subsection{Projektphase 1: Bedarfserhebung}

Die Erhebung des Bedarfs ${ }^{4}$ erfolgte an allen teilnehmenden Hochschulen mittels einer Befragung durch eine externe Agentur. Insgesamt führte sie 22 qualitative Interviews mit verschiedenen Statusgruppen an den Hochschulen. Im Fokus standen Fragen zu Open Science, Open Access und zum Teilen von Forschungsdaten. Darüber hinaus führten die HCU und die HAW zusätzliche Befragungen der eigenen Hochschulmitglieder durch, um ein detaillierteres Bild des Status Quo von Open Science an den jeweiligen Einrichtungen zu erhalten.

Hier wird allerdings nur auf die Befragung an der HCU eingegangen. Diese führte im ersten Halbjahr 2018 qualitative Interviews sowie eine Online-Befragung unter Forschenden und Lehrenden durch. An beiden Befragungsteilen nahmen Personen aller an der HCU vertretenen Statusgruppen und Fachbereiche teil. Die Online-Befragung lehnte sich an den von Christian Heise entwickelten Fragebogen an, ${ }^{5}$ wurde allerdings für den Anwendungszweck leicht modifiziert. Sie umfasste einerseits gekürzte Fragen zum wissenschaftlichen Publizieren, zur Einstellung zu Open Access und Open Data 
sowie zu eigenen Erfahrungen mit dem Teilen von Forschungsergebnissen. Andererseits wurde der Fragebogen um einige HCU-spezifische Aspekte ergänzt (Abfrage von Fachbereich und Status, besondere Wünsche für Services an der HCU).

In einem Pretest mit Projektmitarbeiter*innen wurde der Fragebogen auf Verständlichkeit geprüft. Die Durchführung und Auswertung der Online-Befragung an der HCU erfolgte mit Hilfe der kommerziellen Software ,UniPark‘. Die HCU-Mitglieder wurden per E-Mail zur Teilnahme eingeladen. Aufgrund der geringen Resonanz ( $\mathrm{n}=22$ ) wurden die Ergebnisse nur deskriptiv im Sinne eines Meinungsbildes ausgewertet.

Ergänzend zur Online-Befragung wurden 13 qualitative Interviews geführt. Für diese wurden gezielt Forschende unterschiedlicher Statusgruppen aus den verschiedenen Fachbereichen angesprochen. Die Interviews wurden als teilstandardisierte Leitfadeninterviews geführt, um eine Einheitlichkeit zu gewährleisten, gleichzeitig aber auch den individuellen Gesprächsverlauf berücksichtigen zu können. Der Leitfaden für die Interviews umfasste neben Fragen zu Open Access auch die breitere Thematik Open Science sowie Fragen zu Erfahrungen mit wissenschaftlichem Publizieren und dem Teilen von Forschungsergebnissen. Die Interviews wurden gemäß der Regeln der qualitativen Inhaltsanalyse nach Philipp Mayring $\underline{\underline{6}}$ ausgewertet.

Die Ergebnisse beider Befragungen an der HCU zeigen, dass die Forschenden grundsätzlich eine positive Einstellung zum Thema Open Access haben. Das Schlagwort ist allen bekannt. Als motivierend für die Publikation im OA sehen Forschende vor allem die größere Sichtbarkeit der Veröffentlichungen und damit verbunden die Chance, öfter zitiert zu werden als mit Publikationen, deren Zugang durch Bezahlschranken begrenzt ist. Weitere Vorteile aus Sicht der Forschenden sind der erleichterte wissenschaftliche Austausch und Transfer, das schnellere Publizieren und nicht zuletzt auch die moralische Komponente: Es fühlt sich richtig an, die eigenen Forschungsergebnisse mit allen Interessierten zu teilen.

Die generell positive Einstellung führt aber nicht immer zu tatsächlichen OpenAccess-Publikationen. Die Gründe hierfür sind vielfältig: Zunächst mangelt es vielen Forschenden an tiefergehendem Wissen zur praktischen Umsetzung von Open Access in ihrem Forschungsalltag. So fehlen manchen beispielsweise Kenntnisse darüber, welche Open-Access-Zeitschriften von hoher Qualität und einschlägig für das eigene Fach sind. Zum Teil haben die Befragten auch falsche Vorstellungen und vermuten, dass Veröffentlichungen auf dem institutionellen Publikationsserver nur über die lokale Institutionswebseite auffindbar seien.

Die weiteren Hürden für das Publizieren im Open Access sind zum größten Teil deckungsgleich mit den Ergebnissen anderer Befragungen zum Thema sowohl in

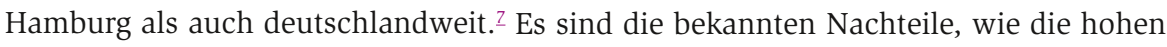
Publikationskosten, die Angst vor Plagiaten, der Mangel an Open-Access-Publikations- 
outlets mit hoher Reputation, sowie die rechtlichen Unsicherheiten bei Zweitveröffentlichungen. Nicht zuletzt ist das Publizieren im Open Access für fast alle Forschenden noch immer vergleichsweise neu. Die Umsetzung ist daher mit erhöhtem Aufwand verbunden, für den die Zeit im Forschungsalltag fehlt. Dementsprechend äußern Forschende den Wunsch, mit spezifischen Services unterstützt zu werden, zum Beispiel bei Open-Access-Zweitveröffentlichungen.

Die Ergebnisse der Befragungen wurden in der weiteren Projektarbeit als Ausgangspunkt genommen, um Maßnahmen zur Förderung von Open Science gezielt auf die Bedarfe der Forschenden auszurichten. In der nächsten Projektphase wurden prototypische Konzepte zur Förderung von Open Science erstellt und erste Ansätze erprobt.

\subsection{Projektphase 2: Prototypische Konzepte}

Ausgangspunkt für die Erstellung der prototypischen Konzepte im Projekt Kulturwandel waren, wie erwähnt, zum einen die Ergebnisse der Befragung. Zum anderen wurden aber auch Handlungsempfehlungen und Berichte über Best Practices aus Veröffentlichungen anderer Institutionen ${ }^{\underline{8}}$ sowie von einschlägigen Webseiten wie ,open-access.net‘ oder dem nationalen Kontaktpunkt Open Access (oa2020-de.de) berücksichtigt. Nicht zuletzt spielte der hochschulübergreifende Austausch im Programm HOS bei der Erstellung der Konzepte eine wichtige zusätzliche Rolle.

Um den Kulturwandel zu Open Access zu unterstützen, bedarf es einer Kombination von Maßnahmen. Die entwickelten Konzepte umfassen daher verschiedene Handlungsfelder. So besteht der prototypische Entwurf der HCU aus fünf Bereichen: Schulungsangeboten, Serviceangeboten, der Förderung von Nachwuchsforschenden, der Einbindung von Multiplikator*innen, sowie dem Orientierungsrahmen an der Hochschule. Für diese Handlungsfelder werden nachstehend Beispiele aus der Umsetzung in den ersten zwei Projektjahren gegeben:

\section{Informations- und Schulungsangebote:}

Ein gutes Beispiel für eine Maßnahme in diesem Handlungsfeld ist eine Reihe mit Veranstaltungen zum Thema Open Science an der HCU, die alle zwei Monate stattfindet. Themen und Informationsmaterialien sind konsequent an den in der Befragung identifizierten Bedarfen der Forschenden ausgerichtet. Aspekte, die die Forschenden besonders interessieren, wie beispielsweise akademische soziale Netzwerke, dienen dabei als Aufhänger, um mit ihnen zusammenhängende 
Informationen zu Open Access zu vermitteln. In der Veranstaltungsreihe wurden verschiedene Formate getestet, von Coffee Lectures über Workshops bis hin zu Filmscreenings. Als besonders erfolgreich erwies sich das Format der Coffee Lecture, das vielfach in Bibliotheken eingesetzt wird. ${ }^{2}$ Einer kurzen Präsentation folgt dabei eine ebenfalls zeitlich eng begrenzte Diskussionsrunde zu einem Thema. Das enge Zeitfenster lässt sich von den Forschenden sehr gut in ihre Tagesplanung integrieren, und sie schätzen den Austausch mit anderen Forschenden in angenehmer Atmosphäre bei Kaffee und Keksen.

\section{Services für die offene Wissenschaft:}

Viele Forschende äußerten in der Befragung den Wunsch nach praktischer Unterstützung, um Open-Access-Veröffentlichungen für sich einfacher realisierbar zu machen. Ein wichtiger Baustein dabei ist die Beratung zu individuellen Fragestellungen, denn nicht jedes Problem lässt sich mit Standardantworten oder -informationen lösen. Zudem kann auf diese Weise ein Kontakt von Forschenden zur Bibliothek aufgebaut bzw. vertieft werden. Ein weiteres Beispiel für dieses Handlungsfeld ist der Aufbau eines Services für Open-Access-Zweitveröffentlichungen. Dieser wird in Zusammenarbeit mit dem Team des Projekts Open-Access-Repositorien von HOS entwickelt.

\section{Nachwuchsförderung:}

Aus Projektsicht besteht gerade bei Nachwuchswissenschaftler*innen die Chance, neue Wege der Kommunikation von Forschungsergebnissen bereits zu Beginn der akademischen Laufbahn ins Bewusstsein zu bringen, bevor sich Routinen in der Forschungspraxis ausgebildet haben. An der HCU sind Schulungsangebote zu Open Access daher in das interne Personalentwicklungsprogramm für Nachwuchsforscher*innen des Forschungsreferats integriert. Darüber hinaus arbeitet die HCU mit der Hamburg Research Academy zusammen, der zentralen Anlaufstelle zur Qualifizierung des akademischen Nachwuchses in Hamburg.

\section{Multiplikatoren und Netzwerke:}

Die Bedeutung von Multiplikator*innen wurde im hochschulübergreifenden Projektteam bereits zu Beginn des Projekts Kulturwandel diskutiert. Es zeigt sich, dass an der HCU auch ganz ohne ein aktives Benennen von ,Open-Science-Botschafter*innen' einzelne Forschende als solche Multiplikator*innen agieren. So werden Informationen über neue entsprechende Services der Bibliothek zum einen in ,Flurgesprächen' weitergegeben, zum anderen geben Teilnehmer*innen von Veranstaltungen die erhaltenen Informationen in ihren Arbeitsgruppen weiter. Über 
diese informellen Formen hinaus, ist im Projekt geplant, positive Beispiele für Open Access aus der Projektlaufzeit aufzubereiten und für die weitere Kommunikation nutzbar zu machen.

\section{Orientierungsrahmen:}

Neben unterstützenden Maßnahmen, die direkt die Kommunikation mit Forschenden betreffen, spielen auch strukturelle Rahmenbedingungen an Hochschulen eine wichtige Rolle bei der Förderung von OA..$^{10}$ Ein Ziel im Projekt Kulturwandel und des Gesamtprogramms HOS ist daher die Erarbeitung einer Open-Access- bzw. Open-Science-Policy für die jeweiligen akademischen Einrichtungen - sofern noch nicht vorhanden. An der HCU wurde ein Entwurf für eine Open-Science-Policy, der auch einen Paragrafen zu Open Access enthält, hochschulweit - auch mit der Hochschulleitung - diskutiert. In diesem Zusammenhang wurden unter anderem Anreizsysteme für Open-Access-Publikationen thematisiert.

Alle genannten Handlungsfelder wurden an der HCU von einer Kommunikationskampagne flankiert, um $\mathrm{OA}$ an der Hochschule stärker ins Bewusstsein zu rücken: Diese begann mit der beschriebenen Befragung als erster Kommunikationsmaßnahme, bei der die Projektmitarbeiter*innen mit den Forschenden ins Gespräch kamen. Weiterhin werden Aktionstage wie die internationale ,Open-Access-Week für eine verstärkte Öffentlichkeitsarbeit genutzt (mit Postern, Flyern und Mailings) und das Projekt Kulturwandel regelmäßig auf Gremiensitzungen vorgestellt. Für die interne Kommunikation haben sich die hochschulweiten E-Mail-Verteiler als vorteilhaft erwiesen, da Informationen auf diesem Weg das gesamte wissenschaftliche Personal erreichen.

Auf Basis der prototypischen Entwürfe und ersten Erfahrungen mit der Umsetzung der entwickelten Maßnahmen wurde vom Team des Projekts Kulturwandel eine Art ,Baukastensystem' für hochschulspezifische Konzepte für Open Science erstellt. Dahinter steht die Erkenntnis, dass sich jede akademische Einrichtung unterscheidet, sei es in der Fächerzusammensetzung, der Größe oder in den Kommunikationsstrukturen. Dementsprechend müssen auch die Ansätze auf diese Besonderheiten zugeschnit-

\begin{tabular}{|c|c|c|}
\hline Bedarfserfassung & $\begin{array}{c}\text { Informations- und } \\
\text { Schulungsangebote }\end{array}$ & Service und Beratung \\
\hline Nachwuchsförderung & $\begin{array}{c}\text { Multiplikatoren und } \\
\text { Netzwerke }\end{array}$ & $\begin{array}{c}\text { Strukturelle } \\
\text { Rahmenbedingungen }\end{array}$ \\
\hline
\end{tabular}

Abb. 3: Kategorien des ,Baukastensystems' für hochschulspezifische Konzepte zur Unterstützung von Open Science. 
ten werden. Das Baukastensystem umfasst sechs Kategorien (s. Abb. 3), die bereits mit Beispielen aus der HCU vorgestellt wurden. Unter jeder Kategorie befindet sich eine Sammlung möglicher Maßnahmen, die als Ideen und Inspiration für die Entwicklung eigener Konzepte genutzt werden können.

\subsection{Projektphase 3: \\ Evaluation und Anpassung von Maßnahmen}

Für das Projekt Kulturwandel ist explizit ein bestimmter Zeitraum für die Pilotphase vorgesehen, in dem Erfahrungen mit Maßnahmen zur Förderung von Open Science gesammelt werden können. Bereits während der Projektlaufzeit findet eine Evaluation dieser Maßnahmen statt, um sie gegebenenfalls anpassen zu können. Im Projekt wurde entschieden, die Zwischenevaluation nicht nur an Kennzahlen wie der Teilnahmeresonanz von Veranstaltungen auszurichten, sondern auch an qualitativen Ergebnissen, wie einem gestiegenen Bewusstsein für Open Science an der Hochschule, das sich auf der Grundlage von Gesprächen ermitteln lässt. In diesem Projektschritt erweist sich einmal mehr die hochschulübergreifende Zusammenarbeit als sehr hilfreich, da der Austausch über gelungene und auch weniger gelungene Maßnahmen essentiell für die Weiterentwicklung der Konzepte ist.

Bei Abfassung dieses Beitrags ist die Projektphase der Evaluation und Anpassung von Maßnahmen noch nicht abgeschlossen. Aus den bisherigen Erfahrungen an der HCU kann aber dennoch eine Zwischenbilanz bezüglich der Erfolgsfaktoren gezogen werden: Wie oben beschrieben, war bereits die Bedarfserfassung insofern ein erster Erfolg, als die hochschulweite Befragung Gelegenheit bot, auf das Thema Open Access aufmerksam zu machen und mit Forschenden direkt ins Gespräch zu kommen. Nicht selten wurde so ein Interview bereits zu einem ersten Beratungsgespräch.

Ein damit zusammenhängender Erfolgsfaktor an der HCU ist die Herstellung eines persönlichen Kontakts zwischen der Bibliothek und den Forschenden sowie die Etablierung fester Ansprechpartner*innen. Das deckt sich mit einem Ergebnis der Befragung durch die Agentur (s.o.): Denn Forschende wünschen sich ein Gesicht hinter einem Service. Das spiegelt sich auch darin wider, dass Anfragen häufiger an persönliche E-Mail-Adressen gerichtet werden als an bestehende Funktionsadressen.

Die Veranstaltungsreihe Open Science trägt als kontinuierliches Angebot weiterhin erfolgreich dazu bei, die Angebote der HCU-Bibliothek zu Open Access bzw. Open Science ins Bewusstsein der Forschenden zu rücken. Hilfreich ist dabei, die Veranstaltungsthemen auf die für die Arbeitswelt der Forschenden relevanten Problemfelder auszurichten. So ist das Thema Zeitschriftenkrise für sich genommen sicher kein 
,Zugpferd' für eine Veranstaltung, kann aber im Zusammenhang mit Hinweisen zur Finanzierung von Open-Access-Publikationen adressiert werden.

Über die bei Veranstaltungen gegebenen Informationen war es an der HCU möglich, bei einigen Forschenden Aha-Erlebnisse zu erzeugen und sie zum Umdenken anzuregen. Die Teilnehmer*innen von Veranstaltungen bzw. Beratungsgesprächen agierten in der Folge gar als Multiplikator*innen. Diese Erfolgsfaktoren hängen sicherlich zum Teil damit zusammen, dass es an der HCU relativ leicht ist, persönliche Kontakte herzustellen und Themen ins Bewusstsein zu bringen. An einer Hochschule mit anderen internen Kommunikationsstrukturen sind unter Umständen auch andere oder zusätzliche Maßnahmen zur Bewusstseinsbildung nötig. Nicht zuletzt ist es gerade die im HOS-Programm vorgesehene institutionsspezifische Anpassung, welche aus Projektsicht zum Erfolg beiträgt.

\section{Ausblick in die Zukunft nach Projektende}

Die letzte, Anfang 2020 noch nicht begonnene Phase im Projekt Kulturwandel soll dazu dienen, dauerhaft tragfähige Konzepte für den Kulturwandel nach Projektende zu entwickeln. Der Kulturwandel bei der Veränderung von tradierten Forschungspraktiken ist ein langfristiger und dauerhafter Prozess, der nicht innerhalb von drei Jahren abgeschlossen sein kann. Zum jetzigen Zeitpunkt lässt sich feststellen, dass durch die Projektarbeit ein Prozess angestoßen wurde, der auch nach 2020 weitergehen wird. An der HCU, die in diesem Beitrag beispielhaft herausgegriffen wurde, ist das Thema Open Access (und übergreifend: Open Science) über die zahlreichen Maßnahmen ins Bewusstsein gerückt. Durch das Projekt wurden Rahmenbedingungen geschaffen, die nach dessen Auslaufen in jedem Fall Bestand haben werden. Es gibt beispielsweise die hochschulweite Diskussion um eine Policy und Anreizsysteme für Open Access bzw. Open Science. Außerdem gibt es Nutzer*innen von Angeboten, die bereits jetzt als Multiplikator*innen agieren. Hochschulübergreifend sind Material- und Wissenssammlungen aufgebaut worden, die nachgenutzt werden können.

Wie die konkrete Planung nach Projektende an den Hamburger Hochschulen aussehen wird, ist immer abhängig von den zur Verfügung stehenden finanziellen und personellen Ressourcen. Im Idealfall enden die einzelnen Phasen des Projekts Kulturwandel - Bedarfserfassung, Erprobung von Maßnahmen, Evaluation und Anpassung - nicht nach einmaligem Durchlauf, sondern entwickeln sich zu einem kontinuierlichen Kreislauf. Denn es gibt stetige Veränderungen, die bei der Unterstützung des Kulturwandels einbezogen werden müssen. Die Arbeit zur Unterstützung einer Kultur des Teilens ist noch lange nicht abgeschlossen. 


\section{Juliane Finger ist Mitarbeiterin im Projekt ,Digitaler Kulturwandel von ,Hamburg Open Science: Zudem ist die promovierte Kommunikations- wissenschaftlerin und studierte Psychologin an der Bibliothek der HafenCity Universität Hamburg für den Bereich Open Access zuständig. Kontakt: juliane.finger@hcu-hamburg.de}

\section{Anmerkungen}

Der Beitrag ist unter der Creative-Commons-Lizenz Namensnennung 4.0 international veröffentlicht. Den Vertragstext finden Sie unter: https://creativecommons.org/licenses/by/4.0/deed.de. Bitte beachten Sie, dass einzelne, entsprechend gekennzeichnete Teile des Werks von der genannten Lizenz ausgenommen sein bzw. anderen urheberrechtlichen Bedingungen unterliegen können.

1 Vgl. Monitor zur Erfassung des Publikationsaufkommens deutscher akademischer Einrichtungen in wissenschaftlichen Zeitschriften. Analysen der Subskriptions- und Publikationsausgaben unterstützen die Transformation in den Open Access, hg. vom Forschungszentrum Jülich, 2020, abrufbar unter: https://open-access-monitor.de (14.08.2020).

2 Zusammen schaffen wir das moderne Hamburg. Koalitionsvertrag über die Zusammenarbeit in der 21. Legislaturperiode der Hamburgischen Bürgerschaft zwischen der SPD, Landesorganisation Hamburg und Bündnis 90/Die Grünen, Landesverband Hamburg, [Hamburg] 2015, S. 47, abrufbar unter: https://www.spd-fraktion-hamburg.de/uploads/tx wfpresse/koalitionsvertrag download.pdf (14.08.2020).

$\underline{3}$ Mehr Informationen über das Programm Hamburg Open Science gibt es auf der Webseite, abrufbar unter https://openscience.hamburg. de (14.08.2020).

4 Ein ausführlicher Bericht über die Ergebnisse der Befragungen im Projekt Kulturwandel wird gesondert veröffentlicht.

$\underline{5}$ Christian Heise, Von Open Access zu Open Science: Zum Wandel digitaler Kulturen der wissenschaftlichen Kommunikation, Lüneburg 2018, abrufbar unter: https://dx.doi.org/10.14619/1303.

6 Philipp Mayring, Qualitative Inhaltsanalyse. Grundlagen und Techniken, 12., überarb. Aufl., Weinheim 2015.

$\underline{7}$ Vgl. z.B. Stefan Drößler, Carsten Elsner, Sybille Hermann, Christian Hoppe, Nicole Rosenke, Markus Weber, Von Bottom up zu Top down. Umfrage: Forschende der Ingenieurwissenschaften erwarten klare Rahmenbedingungen von den Hochschulleitungen bei Open Access und Open Educational Resources, in: o-bib 6 (2019), Nr. 2, S. 80-91, abrufbar unter: https://doi. org/10.25534/tuprints-00010359; vgl. außerdem Heise, Von Open Access (wie Anm. 5).

8 Vgl. Johannes Fournier, Tina Klages, Heinz Pampel (Redaktion), Open-Access-Strategien für wissenschaftliche Einrichtungen: Bausteine und Beispiele, hg. von der Arbeitsgruppe Open Access der Schwerpunktinitiative Digitale Information der Allianz der Deutschen Wissenschaftsorganisationen, Potsdam 2012, abrufbar unter: https://www.bmbf.de/files/openaccess-strategien.pdf (14.08.2020); Madeleine Bondesen, Open Science an der HAW Hamburg - Empfehlungen zur

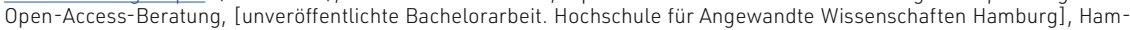
burg 2018; Alessandro Blasetti, Sandra Golda, Dominic Göhring, Steffi Grimm, Nadin Kroll, Denise Sievers, Michaela Voigt, Smash the Paywalls: Workflows und Werkzeuge für den grünen Weg des Open Access, in: Informationspraxis 5 (2019), Nr. 1, abrufbar unter: https://doi.org/10.11588/ip.2019.1.52671.

9 Katrin Ott, Coffee Lectures: Snackable Content für Wissenschaftliche Bibliotheken: Definition, Geschichte und aktuelle Ausprägung [Masterarbeit], Erfurt 2019, online unter: https://doi.org/10.22032/dbt.39370 (14.08.2020).

10 Vgl. Stefan Drößler, Carsten Elsner u.a., Bottom up (wie Anm. 7). 

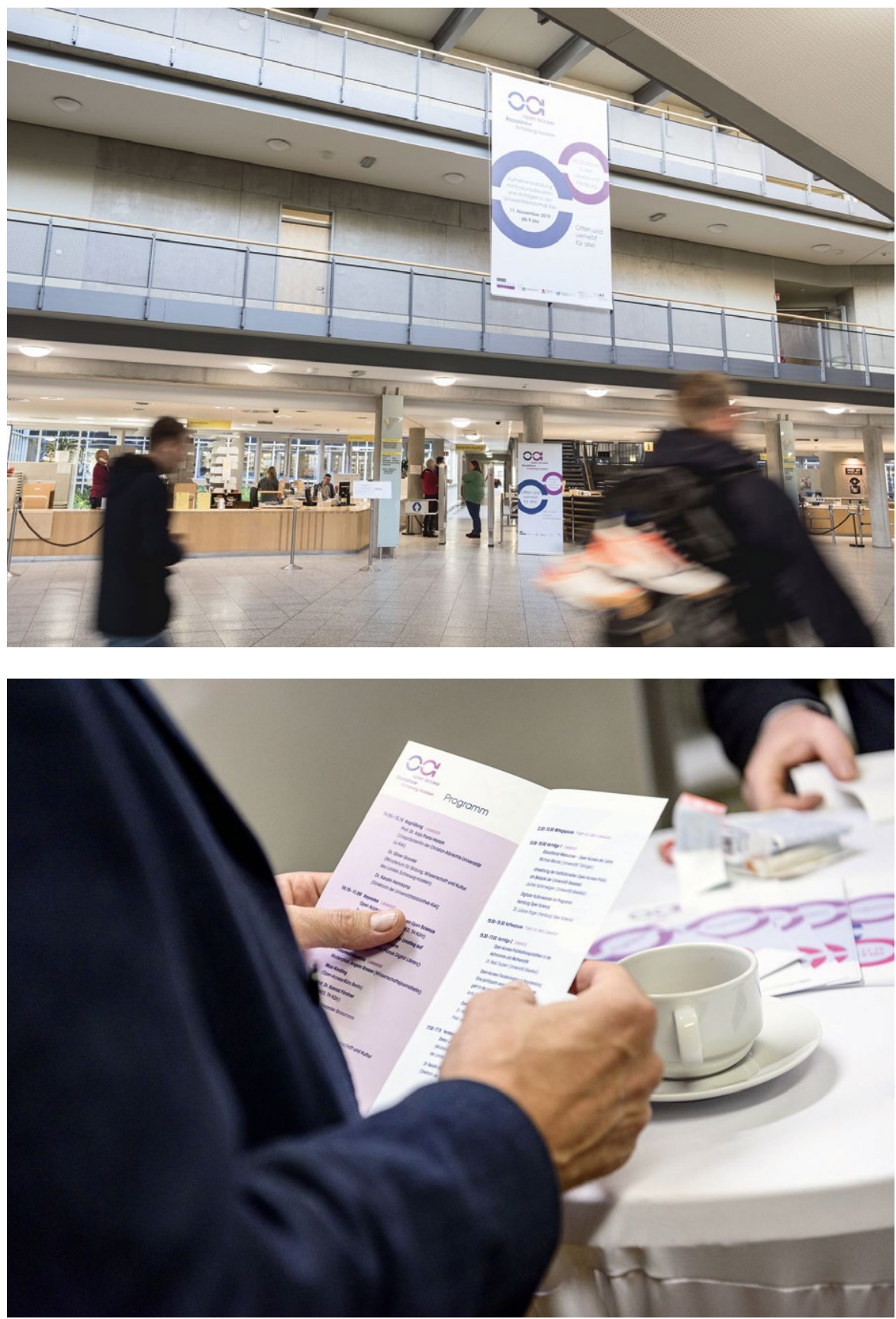